from exposure to antipsychotics. TD is often irreversible, may be debilitating, and cause additional burden to patients with underlying psychiatric conditions.

OBJECTIVE: To assess the impact of developing TD, both with and without other EPS, on healthcare resource utilization (HRU).

METHODS: Data on patients receiving antipsychotics who had schizophrenia, major depressive disorder, or bipolar disorder were extracted from a Medicaid claims database. Patients from the TD cohorts (TD+EPS and TD non-EPS) were matched to those in the non-TD/EPS cohort at $\sim 1: 5$ ratio. HRU outcomes associated with TD were assessed.

RESULTS: TD+EPS $(\mathrm{n}=289)$ and TD non-EPS $(\mathrm{n}=394)$ cohorts were matched with 1398 and 1922 control patients, respectively. The percentage of patients with all-cause and mental disorder-related inpatient admissions increased from baseline to follow-up in the TD+EPS $(12.8 \%$ and $12.5 \%$, respectively) and TD non-EPS (16.0\% and $13.5 \%)$ cohorts, in contrast with slight decreases $(\sim 3 \%)$ in matched controls. A higher percentage of patients in the TD cohorts had medical admissions/visits and claims for drugs that might be used to address TD or EPS than their matched controls at baseline and follow-up. The within-cohort change from baseline to follow-up in the use of potential drugs for TD or EPS was similar between the TD cohorts and their matched controls; however, both TD cohorts exhibited a larger increase in crisis-non-specific psychotherapy services versus matched controls.

CONCLUSIONS: Results demonstrated increased HRU in TD patients with or without other pre-existing EPS, compared with matched controls.

Presented at: Psych Congress; September 16-19, 2017; New Orleans, Louisiana, USA.

FUNDING ACKNOWLEDGEMENTS: This study was funded by Teva Pharmaceutical Industries, Petach Tikva, Israel.

143

Effect of Tardive Dyskinesia on Quality of Life: Patient-Reported Symptom Severity Is Associated With Deficits in Physical, Mental, and Social Functioning

Joseph McEvoy, MD'; Benjamin Carroll, PharmD'; Sanjay Gandhi, PhD ${ }^{2}$; Avery Rizio, PhD ${ }^{3}$; Stephen Maher, PhD ${ }^{3}$; Mark Kosinski, $M A^{3}$; and Jakob Bjorner, $M D, P h D^{3}$

${ }^{1}$ Medical College of Georgia, Augusta, Georgia, USA

2 Teva Pharmaceutical Industries, Frazer, Pennsylvania, USA

${ }^{3}$ Optum, Johnston, Rhode Island, USA
ABSTRACT: Introduction: Tardive dyskinesia (TD), an often-irreversible movement disorder typically caused by exposure to antipsychotics, most commonly affects the face, mouth, and tongue and may be debilitating

OBJECTIVE: To investigate TD burden on patients' quality of life and functionality

METHODS: Adults with clinician-confirmed schizophrenia, bipolar disorder, or major depressive disorder participated in an observational study. Approximately half $(47 \%)$ ofparticipants had a clinician-confirmed TD diagnosis. Participants completed the SF-12v2 Health Survey® (SF-12v2), Quality of Life Enjoyment and Satisfaction Questionnaire Short Form (Q-LES-Q-SF), social withdrawal subscale of the Internalized Stigma of Mental Illness scale (SW-ISMI), and rated the severity of their TD symptoms. Group differences in SF-12v2 physical and mental component summaries (PCS and MCS), Q-LES-Q-SF, and SW-ISMI scores were analyzed.

RESULTS: TD $(n=79)$ and non-TD $(n=90)$ groups were similar in age, gender, and number of patients with schizophrenia, bipolar disorder, and major depressive disorder. TD patients reported significantly worse scores on PCS $(\mathrm{P}=0.003)$, Q-LES-Q-SF $(\mathrm{P}<0.001)$ and SWISMI $(\mathrm{P}<0.001)$ than non-TD patients. The difference in PCS exceeded the established minimal clinically important difference (MCID) of 3 points. When stratified by TD severity, those with more severe symptoms had significantly worse Q-LES-Q-SF $(\mathrm{P}<0.001)$ and SWISMI $(\mathrm{P}=0.006)$ scores than those with less severe symptoms. Differences in PCS $(\mathrm{P}=0.12)$ and MCS $(\mathrm{P}=0.89)$ were in the expected direction and exceeded the MCID.

CONCLUSIONS: Among patients with psychiatric disorders, TD is associated with significant physical health burden and incremental mental health burden. TD severity is also associated with lower overall quality of life and greater social withdrawal.

Presented at: Psych Congress; September 16-19, 2017; New Orleans, Louisiana, USA.

FUNDING ACKNOWLEDGEMENTS: This study was funded by Teva Pharmaceutical Industries, Petach Tikva, Israel.

\section{A Clinical Practice Assessment In Tardive Dyskinesia: Are Physicians Up-to-Date?}

Jovana Lubarda, PhD' ${ }^{\text {; Stacey Hughes }}{ }^{\text {; }}$; and Christoph U. Correll, $M D^{2}$

${ }^{1}$ Medscape Education, New York, NY 
${ }^{2}$ Professor of Psychiatry and Molecular Medicine, Hofstra Northwell School of Medicine, Hempstead, New York; Investigator, The Feinstein Institute for Medical Research; Medical Director, Recognition and Prevention Program, The Zucker Hillside Hospital, Glen Oaks, New York

ABSTRACT: Study Objectives: To assess physicians' current knowledge, skills, competence, and practice barriers regarding tardive dyskinesia (TD) and assess continuing medical education (CME) needs.

ASSESSMENT METHODS: A 29-question clinical practice assessment survey instrument consisting of multiplechoice knowledge and case-based questions was administered online to gather abaseline "snapshot" of knowledge, skills, attitudes, and competence on TD epidemiology, risk factors, diagnosis, current guideline-based management, and emerging management strategies

The survey launched online on a website dedicated to continuous professional development on July 25, 2016, and was made available to healthcare providers without monetary compensation or charge. Data were collected through August 28, 2016

Confidentiality was maintained and responses were de-identified and aggregated prior to analyses

RESULTS: Data were collected for the 1157 psychiatrists and 177 neurologists who responded to all survey questions during the study period. The findings were:

- Epidemiology: $62 \%$ of psychiatrists and $68 \%$ of neurologists were aware that TD affects approximately $20 \%$ of patients treated with neuroleptic agents

- Risk factors: $63 \%$ of psychiatrists and $67 \%$ of neurologists were aware of risk factors for TD, such as older age

- Diagnosis: $93 \%$ of psychiatrists and $71 \%$ of neurologists were aware that Abnormal Involuntary Movement Scale (AIMS) can be used to support diagnosis of TD

- Guidelines: $21 \%$ of psychiatrists and $11 \%$ of neurologists were aware of the American Psychiatric Association guidelines for monitoring of TD, and $56 \%$ of psychiatrists and $42 \%$ of neurologists were aware of the American Academy of Neurology guidelines on treatment of TD

New/emerging treatments: $24 \%$ of psychiatrists and $34 \%$ of neurologists were aware of the mechanisms of action of new/emerging treatments for TD, and $54 \%$ and $44 \%$, respectively, were aware of the clinical data for valbenazine

CONCLUSIONS: This educational research yielded important insights into clinical practice gaps in TD, indicating that both psychiatrists and neurologists would benefit from continuing medical education on epidemiology, risk factors, diagnosis, guideline-based care, and information on how to incorporate new/emerging treatments for TD into practice.

FUNDING ACKNOWLEDGEMENTS: The educational activity and outcomes measurement were funded through an independent educational grant from Neurocrine Biosciences, Inc.

\section{5}

\section{Improving Management of Mood Disorders Through an Online Interactive Patient Case Challenge}

Jovana Lubarda, PhD'; Piyali Chatterjee-Shin'; and Joseph F. Goldberg, $M D^{2}$

${ }^{1}$ Medscape Education, New York, NY

${ }^{2}$ Clinical Professor of Psychiatry, Icahn School of Medicine at Mount Sinai, New York, New York

ABSTRACT: Background: To determine if online continuing medical education (CME) could improve knowledge, competence, and confidence of psychiatrists and primary care physicians (PCPs) in managing patients with major depressive disorder (MDD) and co-occurring hypomanic/manic features.

\section{METHODS:}

- Physicians participated in a 1-hour text-based, online CME activity composed of 2 patient cases with interactive questions related to diagnosis, assessment, and management of various presentations of MDD

- Evidence-based educational feedback was provided following each answer

- Effects of CME were assessed using a repeatedquestion pairs pre- to post-assessment study design where individual participants served as his/her own control

- The assessment included 3 multiple-choice knowledge/competence questions and 1 self-efficacy question that rated confidence in managing MDD with mixed features on a 5-point Likert Scale

- For all questions combined, McNemar's chi-square test assessed the differences from pre- to post-assessment

- $\mathrm{P}$ values measured significance; $\mathrm{P}$ values $<.05$ were considered statistically significant

- Effect size was calculated using Cramer's V by determining the change in proportion of participants who answered questions correctly from pre- to postassessment

- Survey data were collected from December 8th, 2016, to January 24th, 2017. 